\title{
A New Framework for Nonlinear Cointegration Analysis and Its Applica- tion to Finance
}

\author{
Shen Chuanhe, Li Ying* and Feng Liang
}

Institute for Financial Engineering, Shandong Women's University, Jinan, 250300, China

\begin{abstract}
With the purpose of analyzing non-stationary time series, this paper innovates the nonlinear cointegration discriminate analysis by introducing support vector machine and innovated feature-weighting model to overcome existing limitations of two methods, that is, the statistical approach and the neural network used by the nonlinear cointegration theory. Then, the application of the innovated method for the investigation of the connection between financial markets is explored. The empirical analysis demonstrates that the support vector machine is effective in analyzing the money demand function and its stability and has advantages in dealing with nonlinear cointegration relations and estimating nonlinear functions over the two methods above.
\end{abstract}

Keywords: Data mining, Non-stationary time series, nonlinear cointegration analysis, support vector machine, the connection between financial markets

\section{INTRODUCTION}

For the sake of the nonlinear and non-stationary financial time series, many scholars have developed various econometrics models, to tackle effectively, relations among different financial variables. As one of the most important methodologies, the nonlinear cointegration theory, an extension of the basic linear cointegration theory initiated by Granger (1981), Engle \& Granger (1987) [1, 2], and Johansen (1988, 1991) $[3,4]$, has showed powerful capability of depicting the relations. But, discriminate analysis of whether the relations are nonlinear, cointegration constitutes the key step in the nonlinear cointegration test. So far, most literatures address the test, such as Chang \& Park (2003) [5], Lieberman \& Phillips (2008) [6], Choi \& Saikkonen (2010) [7], etc. These methods can be divided in to two categories, that is, the statistical approach and the neural network.

But, all of the two categories above have limitations in nonlinear cointegration test that most of these models widely employ the parametric estimation under the statistics and the nonparametric estimation methods like the neural network, all of which inevitably brought spurious regression phenomena in varying degrees because of their own drawbacks, such as too many strict hypothesis, over fitting, and so on (Karlsen et al., 2007) [8]. Therefore, this paper attempts to propose another test method based on support vector machines, compared with the neural network methodology. In Section 2, the methodology is described to explore multivariables financial time series analysis. Section 3 presents its application to analysis of the connection between the money market and the capital market, with the conclusion in the final section.

\section{NONLINEAR COINTEGRATION ANALYSIS BASED ON SUPPORT VECTOR MACHINES}

The support vector machine is first introduced with the innovated nonlinear cointegration analysis.

\subsection{Support Vector Machines (SVM)}

The SVM is a new generation learning system for small samples and is constructed according to the statistical learning theory by Vapnik (1995) [9], which employs the structural risk minimization principle other than the empirical risk minimization principle generally used by the neural network methodology. It therefore overcomes the shortcomings of the neural network recognition algorithm, such as large samples, "the curse of dimensionality", local optimization, overfitness, etc., and has a better model generalization performance. It is now being established as one of the standard tools for machine learning and data mining, such as pattern recognition, regression analysis and probability density estimation [10]. Here, the support vector regression machine $(\varepsilon-$ SVR hereafter), as one kind of support vector machines, is introduced below [11].

The $\varepsilon$-SVR is involved in the approximation of function, that is, the selection of a special function in an image space through machine learning, with the function expressed as follows:

Given a training set, $\left\{X_{i}, y_{i}\right\}, i=1,2, \ldots, l$, where the input data $X$ is assumed to be a compact domain in a Euclidean space $R^{n}$ and the output data $y$ is assumed to be a closed subset of $R$. Learning from data can be viewed as an approximation of the multivariate function $f(X)$ which represents the relation between the input $X$ and the output $y$. By some nonlinear mapping $\varphi(X)$, the input $X$ is mapped onto a hypoth- 
esis space (or feature space) in which the learning machine (algorithm) selects a certain function $f(X)$.

According to the learning theory, for constructing a nonlinear SVM, the decision function takes the following form:

$$
f(X)=W \cdot \phi(X)+b
$$

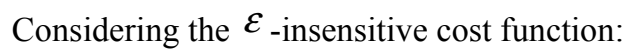

$$
\begin{gathered}
M(y, f(X))=L\left(|y-f(X)|_{\varepsilon}\right) \\
\text { Where, } \quad|y-f(X)|_{\varepsilon}=\left\{\begin{array}{ll}
0, & \text { if }|y-f(X)| \leq \varepsilon \\
|y-f(X)|-\varepsilon, & \text { others }
\end{array},\right.
\end{gathered}
$$

and $\varepsilon$ represents the insensitivity range of the $\varepsilon$-SVR.

This means that the cost is equal to 0 if the deviation of the expected value from the observed value is smaller than the $\varepsilon$.

Then, solving regression problem is equivalent to optimizing the following problem:

$$
\begin{gathered}
\min \frac{1}{2}\|W\|^{2}+C\left(\sum_{i=1}^{l} \xi_{i}^{*}+\sum_{i=1}^{l} \xi_{i}\right) \\
\text { s.t. } y_{i}-\left(W \cdot \varphi\left(X_{i}\right)\right)-b \leq \varepsilon+\xi_{i}^{*}, i=1,2, \cdots, l \\
\left(W \cdot \varphi\left(X_{i}\right)\right)+b-y_{i} \leq \varepsilon+\xi_{i}, i=1,2, \cdots, l \\
\xi_{i}^{*} \geq 0, i=1,2, \cdots, l \\
\xi_{i} \geq 0, i=1,2, \cdots, l
\end{gathered}
$$

Where, $C$ is the regularization constant which plays a trade-off between the regularization performance and the empirical error, and $\xi_{i}, \xi_{i}^{*}$ represent slack variables.

By solving its dual problem, that is, corresponding quadratic programming problem above, the $w$ and $b$ in (1) can be obtained:

$$
\begin{aligned}
& w=\sum_{i=1}^{l}\left(\alpha_{i}^{*}-\alpha_{i}\right) \varphi\left(X_{i}\right) \\
& b=y_{j}-\sum_{i=1}^{l}\left(\alpha_{i}^{*}-\alpha_{i}\right) K\left(X_{i}, X_{j}\right)+\varepsilon
\end{aligned}
$$

The decision function represented in (1) is then transformed into:

$$
f(X)=\sum_{i=1}^{l}\left(\alpha_{i}^{*}-\alpha_{i}\right) K\left(X_{i}, X\right)+b
$$

\subsection{Nonlinear Cointegration Analysis Based on $\varepsilon$-SVR}

The cointegration theory falls into two classes, that is, linear cointegration theory and nonlinear cointegration one. For the linear cointegration theory, the cointegration reflects station degree variability of various non-stationary stochastic series after linear combination. Engle \& Granger (1987) put forward the cointegration notion, which establishes a theory base for us to seek equilibrium relations among two or more non-stationary stochastic series and to set up error correction models for the variables with cointegration relations [2]. What is more, it integrates advantages of short-term and dy- namic models used by time series analysis method to determine the long-term stable relations in econometrics. But, nonlinear cointegration method is being much appreciated because financial time series show the characteristics of strong non-station. Here, only the non-parametric method is discussed and much emphasis will be played on the machine learning approach, such as the SVM. The principal idea is that the $\varepsilon$-SVR is used to improve the discriminate analysis, with the definition of nonlinear cointegration formulated as follows:

If $X$ is a $m \times 1$ order time series vectors and satisfies [4]:

The component of $\left\{X_{i}\right\},\left\{x_{i j}\right\}$, is a long-memory time series (LMT) or $I(1)(i=1,2, \ldots, l ; j=1,2, \ldots, m)$.

If there exists a function $f(\cdot)$, which makes $y_{i}=f\left(x_{i 1}, x_{i 2}, \cdots, x_{i m}\right)$ a zero-mean short memory in mean (SMM) or $I(0)$.

Then, the nonlinear cointegration relation exists among all the component time series of $\left\{X_{i}\right\}$.

Obviously, the estimation of the nonlinear function $f(\cdot)$ becomes the emphasis and the difficulty in nonlinear cointegration analysis, and many methods have been developed to solve this problem so far. Zhang et al. (1999) employed a neural network to test the existence of nonlinear cointegration relation and discussed its feasibility [12]. But, the neural network is still flawed to the discriminate analysis of nonlinear cointegration because of its well-known limitations. Here, the $\varepsilon$-SVR will be applied to overcome these limitations and the specific steps are expressed as follows according to the definition $[1,2]$ :

- Choose financial variable time series as input vectors $X_{i}$ of the $\varepsilon$-SVR, with a known short-memory time series, $\left\{D_{i}\right\}$, used simultaneously as supervised output (i.e. the output vector $\left\{y_{i}\right\}$ ).

- Employ the $\varepsilon$-SVR to estimate the nonlinear function $y_{i}=f\left(x_{i 1}, x_{i 2}, \cdots, x_{i m}\right)$.

- Obtain the estimated output value $\hat{y}_{i}$ using $\hat{y}_{i}=f\left(x_{i 1}, x_{i 2}, \cdots, x_{i m}\right)$ with the $X_{i}$ still the input vector of the $\varepsilon$-SVR.

- $\quad$ Test existence of the nonlinear cointegration relation among component vectors of $\left\{X_{i}\right\}$. This means verifying the long memory characteristic in the time series $\left\{\hat{y}_{i}\right\}$.

If there is still the long memory characteristic in the time series $\left\{\hat{y}_{i}\right\}$, the nonlinear cointegration relation among component vectors of $\left\{X_{i}\right\}$ doesn't exist. Otherwise, this kind of relation is exists.

\section{APPLICATION TO ANALYSIS OF THE CONNECTION BETWEEN FINANCIAL MARKETS}

Actually, the nonlinear cointegration analysis is widely used in financial issues, such as asset pricing (Lieberman \& Phillips, 2008; Amano, et al., 2012) [6, 13]. Here, the nonlinear cointegration analysis based on SVM will be applied 
in the investigation of the connection between financial markets.

As a prevailing method in this field, the cointegration theory is increasingly innovated and the approach based on the $\varepsilon$-SVR above contributes to the innovation. Here, the approach is employed to analyze the connection and the emphasis will aim for a long-term equilibrium relation between the two markets. The main task below is to explore the influence from the capital market on the money demand function and its stability, concerning the changes in influencing factors of the function.

\subsection{The Money Demand Function and its Stability}

The money demand function mainly refers to the direction and extent of effects, from the changes in quantity of money, on the economic process, and its stability is still an existing important problem [14]. The influencing factors of the function can be separated into three categories: the first is scale variables, including wealth and income; the second is opportunity cost variables, like the yield of the asset except for money and the third is other variables, such as asset price risk, monetization of economy, financial structures, etc.

Here, the money demand function can be denoted as follows according to [15]:

$$
\Delta M_{2}=f\left(r_{3-m}, r_{30-y}, r_{\text {bank }}, \Delta_{\text {income }}, \Delta_{\text {index }}^{+}, \Delta_{\text {index }}^{-}\right)
$$

Where, $\triangle M_{2}$ is the change in quantity of money, $M_{2}$; $r_{3-m}, r_{30-y}, r_{\text {bank }}, \Delta_{\text {income }}$ and $\Delta_{\text {index }}$ represent the yield of threemonth treasury, the yield of thirty-year treasury, the interbank money market rates, the percentage variation of nominally disposable income and the percentage variation of stock index respectively.

In the meantime, in order to deeply investigate the changes in demand of the quantity of money caused by the changes in stock index, the $\Delta_{\text {index }}$ is subdivided into two new factors, that is, $\Delta^{+}$index and $\Delta_{\text {index }}^{-}$[16]. The rules for their values are as follows: if the $\Delta_{\text {index }}$ is positive, then the $\Delta^{+}$index is just equal to the $\Delta_{\text {index }}$; otherwise, it is 0 . The formulation is the same for the $\Delta_{i n d e x}^{-}$, which means the $\Delta_{i n d e x}^{-}$is set as the same value as the $\Delta_{\text {index, }}$ and if the $\Delta_{\text {index }}$ is negative; it is 0 .

In fact, the stability of the function, that is, the stability of the relations among variables, is more important than the variables themselves because not only the variables but also their relations vary with economic system, economic structure, financial instruments and financial innovation, etc. All these result in the changes in the function and affect monetary policy efficiency, which deeply reflects the complexity of the function. Therefore, it is necessary that some sophisticated measures should be taken to analyze the complexity, and the nonlinear cointegration method based on the $\varepsilon$-SVR is just for this.

In order to enhance generation ability of the $\varepsilon$-SVR, a method for feature weighting is used here [17]:

Under the notion of feature weighting, the nonlinear mapping $\varphi(X): X \rightarrow \varphi(X)$ in (1) is transformed into:

$$
\varphi_{\theta}(X): X \rightarrow \varphi_{\theta}(X)=\varphi(\theta \cdot X)
$$

Where, $\theta=\left(\theta_{1}, \theta_{2}, \cdots, \theta_{n}\right)$ represents a weight vector for the input vector, which gives the right weight to the right feature.

The dual problem corresponding to (2) becomes:

$$
\begin{aligned}
& \min \quad \frac{1}{2} \sum_{i=1}^{l}\left(\alpha_{i}^{*}-\alpha_{i}\right)\left(\alpha_{j}^{*}-\alpha_{j}\right) K_{\theta}\left(X_{i}, X_{j}\right) \\
& +\varepsilon \sum_{i=1}^{l}\left(\alpha_{i}^{*}+\alpha_{i}\right)-\sum_{i=1}^{l} y_{i}\left(\alpha_{i}^{*}-\alpha_{i}\right) \\
& \text { s.t. } \quad \sum_{i=1}^{l}\left(\alpha_{i}^{*}-\alpha_{i}\right)=0 \\
& \quad 0 \leq \alpha_{i}^{*}, \alpha_{i} \leq C \quad i=1,2, \cdots, l
\end{aligned}
$$

Then, the decision function in (3) is also correspondingly changed into the form:

$$
f(X)=\sum_{i=1}^{l}\left(\alpha_{i}^{*}-\alpha_{i}\right) K_{\theta}\left(X_{i}, X\right)+b
$$

Where, the kernel function in (6) is defined as:

$K_{\theta}\left(X_{i}, X_{j}\right)=K\left(\left(\theta \cdot X_{i}\right),\left(\theta \cdot X_{j}\right)\right)=K\left(\varphi_{\theta}\left(X_{i}\right), \varphi_{\theta}\left(X_{j}\right)\right)$

For example, the radial basis function kernels (RBF) can be written as follows:

$$
K_{\theta}\left(X_{i}, X_{j}\right)=\exp \left(-\sum_{k=1}^{n} \frac{\left(x_{i k}-x_{j k}\right)^{2}}{\sigma_{k}^{2}}\right)=\exp \left(-\sum_{k=1}^{n} \theta_{k}^{2}\left(x_{i k}-x_{j k}\right)^{2}\right)
$$

Where, $\sigma^{2}$ is the width of the radial basis function.

Therefore, it is concluded from (2), (3), (5) and (6) that the feature weighting is equivalent to constructing the kernel function with the same weight vector, $\theta$, as (7), and that this doesn't change the framework and expression of the traditional SVM shown in (2) and (3) (Blum and Langley, 1997; Weston, et al., 2001) [18, 19].

Here, the $\theta$ will be determined by taking the derivative of $f(X)$ in (3) [20]:

$\frac{\partial y_{i}}{\partial x_{i k}}=-\frac{2}{\sigma^{2}} \sum_{j=1}^{l_{s}}\left(\alpha_{j}^{*}-\alpha_{j}\right)\left(x_{i k}-x_{j k}\right) \exp \left(-\frac{1}{\sigma^{2}} \sum_{l=1}^{n}\left(x_{i l}-x_{j l}\right)^{2}\right)$

Thus, the $\theta_{k}$ can be defined through (8) as follows:

$$
\theta_{k}=\frac{1}{M A E}\left[\left(\frac{1}{l} \sum_{i=1}^{l}\left|\frac{\partial y_{i}}{\partial x_{i k}}\right|\right) \cdot\left(\frac{1}{l-1} \sum_{i=1}^{l-1}\left|x_{i}-x_{i+1}\right|\right)\right]
$$

Where, $k=1,2, \ldots, n$, and $M A E$, namely the Mean Absolute Error, represents the prediction performance which is evaluated using the following statistical metrics:

$$
M A E=\frac{1}{l} \sum_{i=1}^{l}\left|y_{i}-f\left(x_{i}\right)\right|
$$


Using (8) and (10), the $\theta_{k}(k=1,2, \ldots, n)$ in (9) can be preliminarily calculated.

\subsection{Empirical Analysis}

Here, the $\triangle M_{2}$ is chosen as the output of the $\varepsilon$-SVR, $y_{i}$, and six influencing factors constitute the input vector, $X_{i}$, including $r_{3-m}, r_{30-y}, r_{\text {bank }}, \Delta_{\text {income }}, \Delta_{\text {index }}^{+}$, and $\Delta_{\text {index }}^{-}$. Therefore, the input vector, $X_{i}$, hence becomes six-dimensional, i.e. $X_{i} \in R^{6}$.

The monthly data for $X_{i}$ and $y_{i}$ is collected from $31 / 8 / 2003$ to $31 / 8 / 2010$, with 84 input vectors and output values obtained respectively, which means $l=84$ in (2).

After the parameters $C$ and sig2 $\left(\sigma^{2}\right)$ are set to 10 and 0.2 respectively, the demand function of money in (4) is obtained after the $\varepsilon$-SVR in (6) is trained by all of $X_{i}$ and $y_{i}$ $(i=1,2 \ldots, 84)$ using MATLAB software.

Now, the nonlinear cointegration analysis based on the $\varepsilon$-SVR is implemented in order to deeply explore whether the long-term stable and balanced relation between the demand quantity of money and the $\Delta_{\text {index }}$ is existent [21]. But, the input vector of the $\varepsilon$-SVR needs to be adjusted before the implementation, which means that the $\triangle M_{2}$ is integrated to $X_{i}$ so that a new $X_{i}$ is obtained, and the $y_{i}$ will be substituted by a supervised time series with zero-mean short memory. The corresponding nonlinear estimation function is thus denoted as follows:

$$
f(\cdot)=f\left(r_{3-m}, r_{30-y}, r_{\text {bank }}, \Delta_{\text {income }}, \Delta_{\text {index }}^{+}, \Delta_{\text {index }}^{-}, \Delta M_{2}\right)
$$

Next, an estimated output value $\left\{\hat{y}_{i}\right\}$ is obtained based on the function $f(\cdot)$, with the new $X_{i}$ as the input vector again. By the nonlinear cointegration discriminant analysis using Eviews statistic software, the $\left\{\hat{y}_{i}\right\}$ is not a long-term memory. Hence, it is concluded that there really exists a nonlinear cointegration relation among the $\Delta M_{2}$ and its influencing factors, such as $r_{3-m}, r_{30-y}, r_{\text {bank }}, \Delta_{\text {income }}, \Delta_{\text {index }}^{+}$, and $\Delta_{\text {index }}^{-}$

And at the same time, these models above are also compared by using the other procedure for efficiency of any model defined by Nash \& Sutcliffe (1970) [22], $R^{2}$, which is a criterion of determining the efficiency of a model in comparison with the average value of data.

$$
R^{2}=1-\frac{\sum_{i=1}^{l}\left(y_{i}-\hat{y}_{i}\right)^{2}}{\sum_{i=1}^{l}\left(y_{i}-\bar{y}\right)^{2}}
$$

Where, $\bar{y}$ is the average value of observed data. This criterion does not indicate the degree of interrelation (namely, correlation coefficient) between estimated and observed values, but it implies the efficiency of the model used. A value of $R^{2}$ close to 1 indicates information for linear dependence agreement between the estimated and observed values. The $R^{2}$ values of different models defined in (12) are shown in Table 1, with their MAE values listed simultaneously.
Table 1. The comparison of $R^{2}$ and $M A E$ values of different models.

\begin{tabular}{|c|c|c|}
\hline & $\boldsymbol{M A} \boldsymbol{E}$ & $\boldsymbol{R}^{2}$ \\
\hline \hline The statistical approach & 0.2133 & 0.7054 \\
\hline The neural network & 0.1651 & 0.8191 \\
\hline The $\varepsilon$-SVR in (3) & 0.1184 & 0.8369 \\
\hline The $\varepsilon$-SVR in (6) & 0.1014 & 0.8633 \\
\hline
\end{tabular}

Table 2. The sensitivity of money demand function to influence factors.

\begin{tabular}{|c|c|c|c|c|c|c|}
\hline & $\boldsymbol{r}_{3-\mathrm{m}}$ & $\boldsymbol{r}_{30-\mathrm{y}}$ & $\boldsymbol{r}_{\text {bank }}$ & $\Delta_{\text {income }}$ & $\Delta_{\text {index }}^{+}$ & $\Delta_{\text {index }}^{\text {ind }}$ \\
\hline \hline+ & 6 & 4 & 9 & 77 & 70 & 11 \\
\hline- & 78 & 80 & 75 & 7 & 14 & 73 \\
\hline Total & 84 & 84 & 84 & 84 & 84 & 84 \\
\hline$A A V$ & 0.19 & 0.30 & 0.25 & 0.27 & 0.58 & 0.40 \\
\hline
\end{tabular}

The $R^{2}$ values of the $\varepsilon$-SVR in (3) and in (6) take 0.8369 and 0.8633 respectively, which shows that the feature weighting $\varepsilon$-SVR outperforms the traditional $\varepsilon$-SVR in the nonlinear cointegration analysis and the former constitutes the best model. Moreover, the $\varepsilon$-SVR both in (3) and in (6) have better advantages in the linear dependence agreement than the neural network because the decision functions represented in (3) and (6) display the linear dependence correlation between the estimated and observed values with the kernel function working in the feature space. Obviously, the selection and option of the kernel function in (3) and (6) will become critical to the decision of the linear dependence agreement, which constitutes an advantage over the other models.

Then, the sensitivity of the function in (11) to its influencing factors can be calculated using (8) and all the values of the sensitivity of the function can be obtained and shown in Table 1, with the symbol "+" standing for the number of positive sensitivity values, "-" the number of negative sensitivity values and " $A A V$ " the Averaged Absolute Value of all the sensitivity values. Therefore, the $A A V$ of the $K^{\text {th }}$ component of $X_{i}$ can be expressed as follows Table 2:

$$
A A V=\frac{1}{l}\left|\frac{\partial y_{i}}{\partial x_{i k}}\right|
$$

Then, the structure of interaction between the money demand function and every influencing factor, reflecting shortterm changes in their direction and extent to which the function varies correspondingly, can be found through the analysis of $A A V$ in (13) and the results are all shown in Table 2.

Obviously, it is shown in Table 2 that in all influencing factors, only $\Delta^{+}{ }_{\text {index }}$ and $\Delta_{\text {index }}^{-}$display unstable interaction with the demand quantity of money. 
Table 3. The comparation of model performation between the support vector machine and other approaches.

\begin{tabular}{|c|c|c|}
\hline & $\boldsymbol{\alpha}=\mathbf{5 \%}$ & $\boldsymbol{\alpha}=\mathbf{1 0 \%}$ \\
\hline \hline The statistical approach & 1.2734 & 1.2148 \\
\hline The neural network & 1.2916 & 1.2478 \\
\hline The $\varepsilon$-SVR in (3) & 1.2873 & 1.2167 \\
\hline The $\varepsilon$-SVR in (6) & 1.2826 & 1.2136 \\
\hline
\end{tabular}

Now, the nonlinear cointegration analysis based on the improved $\varepsilon$-SVR is implemented in order to deeply explore whether the long-term stable and balanced relation between the demand of the quantity of money and the $\Delta_{\text {index }}$ exists.

Next, an estimated output value $\left\{\hat{y}_{i}\right\}$ is obtained based on the function $f(\cdot)$, with the new $X_{i}$ as the input vector again. By the nonlinear cointegration discriminate analysis using Eviews statistic software, the $\left\{\hat{y}_{i}\right\}$ is not a long-term memory. Hence, it is concluded that there really exists a nonlinear cointegration relation among the $\triangle M_{2}$ and its influencing factors, such as $r_{3-m}, r_{30-y}, r_{\text {bank }}, \Delta_{\text {income }}, \Delta_{\text {index }}^{+}$, and $\Delta^{-}{ }_{\text {index. }}$

The empirical results based on other nonlinear cointegration discriminate analysis methods are also carried out so that their performance can be compared. The corresponding modified $R / S$ values under the statistical value $0.4<\mathrm{p}<0.5$ is shown in Table 3.

It is not difficult to see that for the different conference degree $\alpha=5 \%$ and $\alpha=10 \%$, the modified $R / S$ values in the $\varepsilon$-SVR in (3) and (6) are all more adjacent to those in the statistical approach than that in the neural network, with the $\varepsilon$-SVR in (6) outperforming the $\varepsilon$-SVR in (3). This means that the $\varepsilon$-SVR can seize the nonlinear relations among the $\triangle M_{2}$ and its influencing factors and the improved $\varepsilon$-SVR in (6) exhibits its advantages. This demand that the monetary policymakers should pay close attention to the changes in stock market so that the monetary policy can preferably reflect the impact from the stock prices.

\section{CONCLUSION}

The nonlinear analysis methods are continuously emerging and the research towards their analysis mechanism of the nonlinear cointegration is deepening day by day. As a kind of effective artificial intelligence technology, the support vector machine is playing increasingly important role in the innovation of nonlinear cointegration analysis which is just demonstrated in empirical analysis. Compared with the neural network already used in the nonlinear cointegration analysis, the support vector machine shows more satisfactory performance in model generation and will be investigated in the selection and option of the kernel function and in the self-adaptive adjustment of the model structure to the economic variables.

And as for the application to the analysis of the connection between financial markets, the next work will be con- centrated on analyzing the high-order effects of input variables and capturing the uncertain volatility in the states of the economy, which will deeply improve the robustness of the model proposed.

\section{CONFLICT OF INTEREST}

The authors confirm that this article content has no conflict of interest.

\section{ACKNOWLEDGEMENTS}

The work is simultaneously sponsored by the National Social Science Fund Projects of China (NO. 13BJY026), the National Statistical Science Research Program (NO. 2012LY054) and Shandong Province Natural Science Foundation (NO. ZR2012GM006).

\section{REFERENCES}

[1] C.W.J. Granger, "Some properties of time series data and their use in econometric model specification", J. Economet., vol. 16, pp. $121-130,1981$

[2] R. Engle and C.W.J. Granger, "Cointegration and error correction: representation, estimation and testing", Econometrica, vol. 55, pp. 251-276, 1987.

[3] Johansen S., "Statistical analysis of cointegration vectors", J. Econ. Dyn. Control., vol. 12, pp. 231-254, 1988.

[4] S. Johansen, "Estimation and hypothesis testing of cointegration vectors in Gaussian vector autoregressive models", Econometrica, vol. 59, pp. 1551-1580, 1991.

[5] Y. Chang and J.Y. Park, "Index models with integrated time series", J. Econ., vol. 114, pp. 73-106, 2003.

[6] O. Lieberman and P.C.B. Phillips, "Refined inference on long memory in realized volatility", Economet. Rev., vol. 27, pp. 254267, 2008.

[7] I. Choi and P. Saikkonen, Tests for nonlinear cointegration. Cambridge: Econometric Theory-Cambridge University Press, 2010.

[8] H.A. Karlsen, T. Myklebust, and D. Tjøstheim, "Nonparametric estimation in a nonlinear cointegration type model", Ann. Stat., vol. 35, pp. 252-299, 2007.

[9] V. Vapnik, The nature of statistical learning theory. New York: Springer, 1995.

[10] N. Cristianini and J.S. Taylor, Support vector machines and other kernel-based learning methods. Cambridge: Cambridge University Press, 2000.

[11] V. Vapnik, S.E. Golowich, and A. Smola, "Support vector method for function approximation, regression estimation, and signal processing", in Advances in Neural Information Processing Systems 9, M. Mozer, M. Jordan and T. Petsche, Eds. Cambridge, MA: The MIT Press, pp. 281-287, 1997.

[12] S.Y. Zhang, Q.F. Xu and H. Zhou, Financial time series analysis. Beijing: Tinghua university press, 2008 [in Chinese].

[13] T. Amano, T. Kato, and M. Taniguchi, "Statistical estimation for CAPM with long-memory dependence”, Adv. Dec. Sci., vol. 2012, pp. 1-12, 2012.

[14] H. Johnson, "Monetary theory and policy", Am. Econ. Rev., vol. 52, pp. 335-384, 1962.

[15] M. Friedman, "Money and the stock market", J. Polit. Econ., vol. 96, pp. 221-245, 1988.

[16] G. Li, "A new challenge from the development of the capital market to the monetary policy", Graduate School Chinese Academy of Social Sciences, 2001 [in Chinese].

[17] C.H. Shen, X.R. Wang, and D. Yu, "Feature weighting of support vector machines based on derivative saliency analysis and its application to financial data mining", Int. J. Adv. Comput. Technol., vol. 4, pp. 199-206, 2012.

[18] A.L. Blum and P. Langley, "Selection of relevant features and examples in machine learning", Artif. Intell., vol. 97, pp. 245-271, 1997. 
[19] J. Weston, S. Mukherjee, O. Chapelle, M. Pontil, T. Poggio and V. Vapnik, "Feature selection for SVMs. Lection for SVMs", In: $A d-$ vances in Neural Information Processing Systems 13, Sara A. Solla, Todd K. Leen and Klaus-Robert M"uller, eds. MIT Press, Cambridge, MA, 2001.

[20] L.J. Cao, K.S. Chua, Q.M. Gu and H.P. Lee, "Saliency analysis of support vector machines for gene selection in tissue classification", Neural Comput. Appl., vol. 11, pp. 244-249, 2003.
[21] V. Vapnik, A. Vashist and N. Pavlovitch, "Learning using hidden information (learning with teacher)", in Proceedings of the 2009 international joint conference on Neural Networks, Atlanta, Georgia, USA, pp. 3188-3195, 2009.

[22] J.E. Nash and J.V. Sutcliffe, "River flow forecasting through conceptual models, Part I: A discussion of principles", J. Hydrolog., vol. 10 , pp. 282-290, 1970.

Received: September 22, 2014

(c) Chuanhe et al.; Licensee Bentham Open.

This is an open access article licensed under the terms of the Creative Commons Attribution Non-Commercial License (http://creativecommons.org/licenses/by-nc/3.0/) which permits unrestricted, non-commercial use, distribution and reproduction in any medium, provided the work is properly cited. 